\title{
Influenza: one or two more questions Miranda Robertson
}

When we asked Peter Doherty to write a question-and-answer piece on influenza [1], Australia, where he is based, had one reported case of influenza A (H1N1). At the time of writing this editorial, Australia has more than 1,200 cases (though to date no deaths) and has triggered the announcement by WHO of a global pandemic.

Received wisdom has it that pathogens are not generally lethal to the hosts they normally infect, because they could not survive if they were. Pathogenicity thus becomes adapted to a level at which the host survives to become reinfected (or to produce young that become infected). The most notable example of such adaptation is in the herpesviruses, which have evolved a quite extraordinary repertoire of devices for avoiding human immunity and with which most human adults in the Western world are chronically infected. Herpesviruses persist through latency. Influenza virus belongs to a different strategic class, which proliferates rapidly and escapes in coughs and sneezes, leaving the host immune. Most humans survive infection with human influenza viruses; but the adaptive truce may break down when the human viruses recombine with viruses of avian or swine origin: hence the high human mortality associated with the H5N1 avian influenza virus that emerged into public consciousness in 2005 . The so-called swine H1N1 influenza virus that is the cause of the current pandemic is apparently a triple- reassortant, with genes of swine, human and avian origin [2,3]. Unlike $\mathrm{H} 5 \mathrm{~N} 1$ it is readily transmissible between humans, but it seems - so far at least - otherwise less uncouth, and in most people causes only mild disease; so perhaps in respect both of transmissibility and of pathogenicity it reflects its human rather than its swine or avian origins. What makes this virus particularly dangerous, as Peter Doherty and Stephen Turner explain in their Q\&A in this issue of Journal of Biology [1], is simply that most of us are not immune to it, and it was not, until now, on the agenda for inclusion in the seasonal influenza vaccine programme.

It is probably the level - or rather the distribution - of population immunity that also partly accounts for the atypical pattern of mortality of pandemic as against the usual seasonal influenza. Whereas seasonal influenza is more likely to kill the old, pandemic influenza (including the present $\mathrm{H} 1 \mathrm{~N} 1$ influenza) tends preferentially to kill the young. This is thought to be because older individuals are likely to have some level of immunity due to crossprotective antibodies - that is, antibodies against similar features of other, in this case past, influenza viruses [4]. (I ought however to restate that disease due to influenza A (H1N1) seems generally mild; and indeed mortality is almost certainly even lower than it seems, because it is highly likely that many infected individuals never bother to consult a doctor and the number of people actually infected therefore probably substantially exceeds the number reported.)

The options for vaccine development are discussed by Doherty and Turner; but for the coming (or in the Southern hemisphere, current) flu season, the traditional methods will have to suffice. Among the problems in developing current influenza vaccines, notoriously, is the tendency of the virus to mutate rapidly so that variants can escape detection by otherwise immune individuals (see [1]). Influenza A (H1N1) however seems relatively homogeneous antigenically (see [3]), and the main obstacle to rapid manufacture of the large number of doses that will be required for effective population protection is the painfully cumbersome method by which influenza vaccines are produced (see [1]). Hence the interest in new adjuvants.

An adjuvant, in immunology, is a substance that increases the strength of an adaptive immune response. Adaptive immune responses are immune responses focused on molecular features specific to a given pathogen (operationally defined as antigens) and that are induced by exposure and confer lasting immunity. The induction of adaptive immunity is the basis for vaccination, and all currently effective vaccines work by inducing the production of antibodies (although antibodies are not the only effector mechanism of the adaptive immune response: see [1]). 
The use of an adjuvant could greatly reduce the amount of vaccine needed, and new adjuvants are being tested for use in influenza vaccines.

How do adjuvants work? In principle, the answer is clear and biologically elegant [5]. Adaptive immune responses depend upon the selective activation and proliferation of circulating lymphocytes bearing receptors that recognize specific antigens, including, but not exclusively, molecules expressed by pathogens. Lymphocytes however cannot be activated by antigen alone, and require a second signal which is delivered by cells of the innate immune system, which recognize conserved molecular features common to all members of particular classes of pathogens. The innate immune system thus focuses adaptive immunity on pathogens. Adjuvants activate the innate immune system. The molecular mechanism of this adjuvant effect is understood for inflammatory responses of the adaptive immune system, which are invoked by a family of receptors (TLRs) that are expressed by innate immune cells and recognize a wide range of distinctive features of pathogens.

TLR ligands however are too dangerous for use in human vaccines (because of the inflammatory responses they invoke), and for many vaccines adjuvants are unnecessary, because the killed organisms themselves have intrinsic adjuvant activity - that is, they themselves have features that activate innate immunity. This is the case for influenza virus in current vaccine preparations. Where adjuvant is necessary, either because intrinsic activity is too low, or in vaccines based on purified components that have lost their adjuvanticity in purification, the only adjuvant currently in use in human vaccines is alum - a general term for salts of aluminium, which have been in use in human vaccines since early in the $20^{\text {th }}$ century and that invoke good antibody responses. How does alum work? We do not know. New adjuvants in preparation for use in influenza vaccines include oil-inwater emulsions; we do not know how they work either.

It is known that distinct types of pathogen tend to evoke distinct kinds of adaptive immune response both different kinds of cell and different kinds of antibody specialized to eliminate pathogens with different properties and methods of invasion and proliferation. Protection from influenza virus depends, as Doherty and Turner explain, on neutralizing antibodies that prevent the virus from entering cells. Recent evidence implicates intracellular components of the innate immune system, which are being recognized in increasing numbers, in the adjuvant effects of alum (see [5]). So it seems likely that the riddle of the adjuvanticity of alum, and of the oil-and-water emulsions that may be deployed in influenza vaccines, once solved, will answer some important outstanding questions about how innate immune responses fine-tune adaptive ones.

Miranda Robertson, Editor

editorial@jbiol.com

\section{References}

I. Doherty PC, Turner SJ: Q\&A: What do we know about influenza and what can we do about it? J Biol 2009, 8:46.

2. Trifinov V, Khiabanian H, Rabadan R: Geographic Dependence, Surveillance, and Origins of the 2009 Influenza A (HINI) Virus. N Engl J Med 2009, in press.

3. Garten RJ, Davis CT, Russell CA, Shu $B$, Lindstrom S, Balish A, Sessions WM, Xu X, Skepner E, Deyde V, OkomoAdhiambo M, Gubareva L, Barnes J, Smith CB, Emery SL, Hillman MJ, Rivailler P, Smagala J, de Graaf M, Burke DF, Fouchier RA, Pappas C, Alpuche-Aranda CM, López-Gatell H, Olivera H, López I, Myers CA, Faix D, Blair PJ, Yu C et al:: Anitegenic and Genetic Characteristics of SwineOrigin 2009 A(HINI) Influenza Viruses Circulating in Humans. Science 2009, in press.

4. Miller MA, Viboud C, Balinska M, Simonsen L: The Signature Features of Influenza Pandemics - Implications for Policy. N Engl J Med 2009, in press.

5. McKee AS, Munks MW, Marrack P: How do adjuvants work? Important considerations for the new generation of adjuvants. Immunity 2007, 27:687690.

Published: 12 June 2009

Journal of Biology 2009, 8:45

(doi:10.1 186/jbioll58)

The electronic version of this article is the complete one and can be found online at http://jbiol.com/content/8/5/45

(c) 2009 BioMed Central Ltd 